Arq. Bras. Med. Vet. Zootec., v.71, n.2, p.447-454, 2019

\title{
Avaliação de dois testes sorológicos comerciais para diagnóstico das infecções pelo FIV e pelo FeLV
}

[Evaluation of two point-of-care tests to diagnosis of FIV and FeLV infections]

\author{
S.O. Medeiros ${ }^{1}$, B.J.A. Silva ${ }^{2}$, A.L. Carneiro ${ }^{3}$, O.C. Ferreira Júnior ${ }^{1}$, A. Tanuri ${ }^{1}$ \\ ${ }^{1}$ Universidade Federal do Rio de Janeiro - Rio de Janeiro, RJ \\ ${ }^{2}$ Centro de Apoio e Diagnóstico Veterinário - Rio de Janeiro, RJ \\ ${ }^{3}$ Aluno de graduação - Universidade Castelo Branco - Rio de Janeiro, RJ
}

S.O. Medeiros1
https:///orcid.org/0000-0001-5492-1532
B.J.A. Silva2
https:///orcid.org/0000-0002-0362-1860
A.L. Carneiro3
https://orcid.org/0000-0002-8572-060X
O.C. Ferreira Júnior1
https://orcid.org/0000-0002-1970-8936
A. Tanuri1
https://orcid.org/0000-0003-0570-750X

\section{RESUMO}

FIV e FeLV são retrovírus associados principalmente com neoplasias. Dois testes rápidos são disponibilizados no Brasil para o diagnóstico dessas infecções: um kit de imunocromatografia de fluxo bidirecional (SNAP ${ }^{\circledR}$ Combo IDEXX) e um kit de imunocromatografia de fluxo lateral unidirecional (ALERE/BIONOTE Anigen Rapid). O objetivo deste estudo foi comparar o teste SNAP ${ }^{\circledR}$ com o teste ALERE. Amostras de sangue de 178 gatos foram testadas utilizando-se ambos os kits. A reação em cadeia de polimerase em tempo real (qPCR) foi empregada como método confirmatório para todos os resultados. O teste $\mathrm{SNAP}^{\circledR}$ apresentou sensibilidade e especificidade de $100 \%$ para FIV; a sensibilidade e a especificidade do teste ALERE foram de $96,15 \%$ e 98,68\%, respectivamente. A sensibilidade e a especificidade para o FeLV foram de 93,02\% e 96,30\% para o teste SNAP ${ }^{\circledast}$ e de $90,70 \%$ e $97,78 \%$ para o teste ALERE. Ainda em relação ao FeLV, três amostras com resultado positivo na $\mathrm{qPCR}$ obtiveram resultado falso-negativo em ambos os testes. Não houve diferença estatisticamente significante entre os métodos. Considerando a qPCR como padrão-ouro, o teste SNAP ${ }^{\circledast}$ apresentou maior sensibilidade e especificidade para o FIV, e o teste ALERE apresentou maior especificidade para o FeLV. Os resultados mostraram uma boa correlação entre os testes.

Palavras-chave: retrovírus felino, FIV, FeLV, diagnóstico sorológico

\section{ABSTRACT}

FIV and FeLV are Retrovirus associated mainly with feline neoplasms. Two point-of-care tests are commercially available in Brazil for diagnosis of these infections: a bidirectional flow immunochromatography kit (IDEXX SNAP ${ }^{\circledR}$ Combo) and a lateral unidirectional flow immunochromatography kit (ALERE/BIONOTE Anigen Rapid). The aim of this study was to compare SNAP ${ }^{\circledR}$ and ALERE tests. Blood samples obtained from 178 cats were evaluated using both tests. Quantitative real-time polymerase chain reaction (qPCR) was used as confirmatory test for all samples. The sensitivity and specificity of SNAP ${ }^{\circledR}$ test was $100 \%$ for FIV, and for ALERE test was $96.15 \%$ and $98.68 \%$, respectively. The sensitivity and specificity for FeLV was $93.02 \%$ and $96.30 \%$ for SNAP ${ }^{\circledR}$ test and $90.70 \%$ and $97.78 \%$ for ALERE test. Three samples with a qPCR positive result for FeLV obtained a false negative result in both SNAP ${ }^{\circledR}$ and ALERE tests. There was no statistically significant difference between the two methods. Considering qPCR as gold standard method, the SNAP® test showed higher sensitivity and specificity for FIV, and the ALERE test presented higher specificity for FeLV. The results showed good agreement among the tests.

Keywords: feline retrovirus, FIV, FeLV, serological diagnosis

\section{INTRODUÇÃO}

O vírus da leucemia felina (FeLV) e o vírus da imunodeficiência felina (FIV) são vírus envelopados, pertencentes à família Retroviridae e aos gêneros Gammaretrovirus e Lentivirus, respectivamente. A família Retroviridae se diferencia das demais famílias por sua forma de

Recebido em 7 de junho de 2017

Aceito em 2 de julho de 2018

E-mail: sheila.omedeiros@gmail.com replicação, que ocorre em parte no citoplasma e em parte no núcleo da célula hospedeira. O RNA do vírus é retrotranscrito em uma fita dupla de DNA, chamada DNA complementar (cDNA), pela ação da enzima transcriptase reversa e, posteriormente, esse cDNA será inserido no genoma da célula hospedeira, sendo, então, denominado provírus ou DNA proviral. A transcrição do provírus dá origem a um RNA 
mensageiro viral, que será utilizado na síntese de proteínas virais e na formação de uma nova partícula infecciosa (Ravazzolo e Costa, 2007).

A principal via de transmissão de ambos é a saliva, porém a mordedura é o meio mais eficaz de transmitir o FIV (Matteucci et al., 1993), enquanto a lambedura é mais importante na transmissão do FeLV (Gomes-Keller et al., 2006). A transmissão também pode ocorrer por meio do leite materno, do sêmen, por via transplacentária, perinatal e por transfusão sanguínea (Hosie et al., 2009). Os machos adultos com acesso à rua são considerados o grupo de risco devido ao comportamento da espécie (Hartmann, 2012).

Os sinais clínicos e as doenças relacionadas aos vírus podem variar, no entanto a infecção pelo FeLV aumenta em mais de 60 vezes o risco de desenvolvimento de neoplasias, especialmente o linfoma e as leucemias (Hartmann, 2012). Já para o FIV, doenças crônicas e/ou infecções oportunistas são comumente observadas (Arjona et al., 2000).

Após a exposição ao FeLV, existem diferentes possibilidades de evolução da infecção viral, podendo a doença ser classificada em quatro fases: progressiva, regressiva/latente, focal e abortiva. Na infecção progressiva, ocorre a disseminação viral, com o desenvolvimento de doenças linfoproliferativas ou mieloproliferativas (Torres et al., 2005). Na infecção regressiva/latente, os anticorpos controlam a viremia após algumas semanas e o vírus passa a não ser detectado no sangue periférico, embora o DNA proviral esteja presente nas células. $\mathrm{Na}$ infecção focal ou atípica, o vírus se replica apenas em algum tecido ou órgão, e na infecção abortiva o felino exposto ao vírus consegue responder efetivamente à infecção. Essas duas últimas formas da infecção são menos comuns (Hofmann-Lehmann et al., 2008).

Assim como no caso do FeLV, a infecção pelo FIV é dividida em fases: aguda, assintomática e fase aids. Na fase aguda, os animais podem apresentar um pico febril com linfadenomegalia; logo em seguida, a resposta imune controla parcialmente a carga viral e o felino entra na fase assintomática, que dura vários anos. Apesar da ausência de sintomas, a queda progressiva de linfócitos T CD4+ e a inversão da relação
CD4:CD8 levam à fase aids, que é caracterizada por sinais clínicos referentes a doenças crônicas (Obert e Hoover, 2002). A fase aids é caracterizada por uma alta carga viral, com sinais clínicos mais graves, devido a infecções secundárias e oportunistas (Diehl et al., 1996).

O diagnóstico dessas infecções é importante devido à gravidade das consequências decorrentes delas e pelo fato de que esse conhecimento irá influenciar no manejo do animal e no tratamento adequado. A American Association of Feline Practitioners (AAFP) recomenda que o diagnóstico realizado com teste de triagem seja confirmado com outra metodologia. O teste rápido (TR) é utilizado para o diagnóstico de ambas as infecções como metodologia de triagem, permite obter resultado em 10 minutos e está disponível para detecção dos dois vírus simultaneamente, utilizando soro, sangue total ou plasma. O diagnóstico para FeLV é feito por meio da detecção da proteína p27 do capsídeo; já para o FIV, o melhor marcador da infecção é a detecção de anticorpos da classe IgG (Levy et al., 2008). Devido à simplicidade e rapidez de execução, esses testes são comumente utilizados na rotina clínica e laboratorial. Já a reação em cadeia da polimerase (PCR) convencional ou em tempo real (qPCR) é uma metodologia molecular capaz de detectar o material genético do vírus no sangue periférico, sendo indicada como teste confirmatório para ambas as infecções (Westman et al., 2016).

Um fator complicador no diagnóstico do FeLV é que nem todas as fases são detectadas pelos métodos sorológicos. Nas infecções regressiva/latente, focal e abortiva, o antígeno p27 não está circulante, e esses felinos apresentam resultado falso-negativo nos testes sorológicos. Nessas situações, a única metodologia capaz de identificar o DNA proviral é a PCR (Hofmann-Lehmann et al., 2008). Em relação ao FIV, os anticorpos apresentam níveis detectáveis cerca de quatro semanas após a infecção (Hosie et al., 2009). A detecção sorológica desses anticorpos pode apresentar resultado falso-negativo na fase de janela imunológica e na fase aids (Dandekar et al., 1992); neste caso, pela disfunção imunológica que ocorre na fase final da infecção (Paillot $e t$ al., 2005). Já os resultados falso-positivos podem ser encontrados principalmente em filhotes de até seis meses de vida, pela detecção 
dos anticorpos maternos adquiridos pela amamentação (Hosie et al., 2009).

No mercado brasileiro, existem dois TRs disponíveis para o clínico: o SNAP $^{\circledR}$ Combo FeLV Ag/FIV Ab Test IDEXX (imunocromatografia de fluxo bidirecional) e o ALERE/BIONOTE FIV Ac/FeLV Ag Test Kit (imunocromatografia de fluxo lateral unidirecional), conhecido internacionalmente como Anigen Rapid Test. Ambos os kits detectam o antígeno p27 do FeLV e anticorpos da classe IgG anti-FIV, porém, no caso do SNAP $^{\circledR}$, os anticorpos são direcionados para os antígenos p15 e p24, e no teste ALERE para os antígenos p24 e gp40 do FIV (Westman et al., 2015). A sensibilidade e a especificidade do teste SNAP $^{\circledR}$ para o FeLV são de $98,6 \%$ e $93,5 \%$, respectivamente; já o teste ALERE possui sensibilidade e especificidade de $100 \%$ para o FeLV. Para o FIV, a sensibilidade do teste
SNAP $^{\circledR}$ é de $93,5 \%$ e a especificidade é de $100 \%$. A especificidade do teste ALERE para o FIV é de $98 \%$ e a sensibilidade é de $96 \%$ (bula dos kits).

O método imunocromatográfico de fluxo unidirecional (ALERE) foi recentemente introduzido no mercado brasileiro, sendo necessário avaliar sua equivalência em relação ao kit já existente $\left(\mathrm{SNAP}^{\circledR}\right)$. O objetivo deste trabalho foi comparar dois TRs comerciais para detecção de FIV e FeLV.

\section{MATERIAL E MÉTODOS}

Foram avaliados os dois TRs disponíveis no mercado, o SNAP ${ }^{\circledR}$ Combo e o teste ALERE, em um total de 178 amostras de sangue de felinos domésticos (Felis silvestris catus, Linnaeus, 1758). O resultado desses testes é visualizado pela alteração de cor na janela de resultados (Fig. 1).

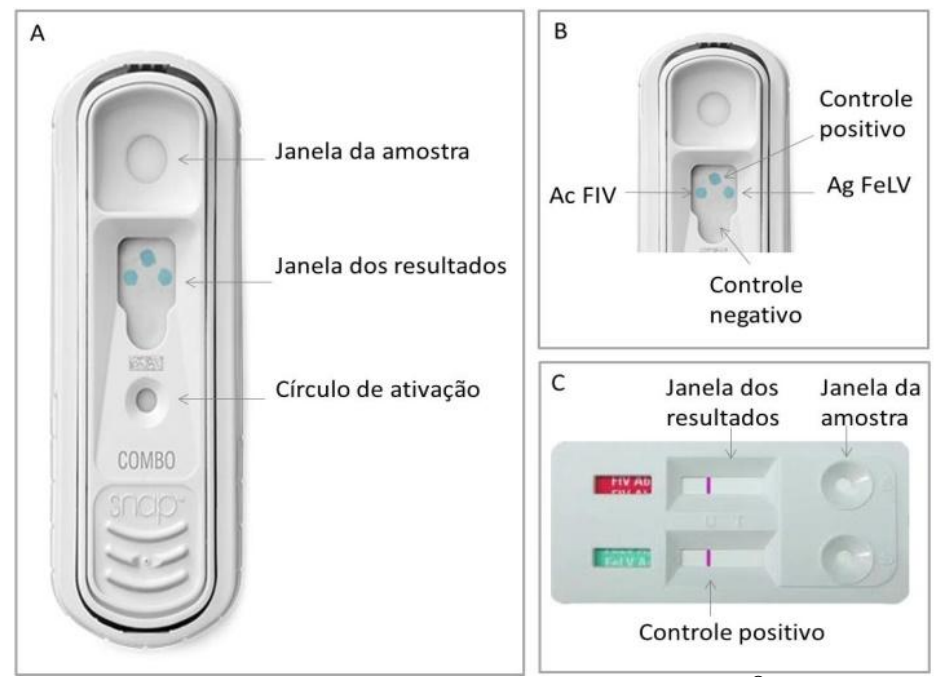

Figura 1. Apresentação dos testes avaliados: (A) Dispositivo SNAP ${ }^{\circledR}$ combo, mostrando a janela de amostra, a janela de resultados e o círculo de ativação; (B) Aproximação da janela de resultado do kit SNAP $^{\circledR}$, mapeando as áreas onde os resultados são observados após a adição da amostra; (C) Kit ALERE - janela de amostra e janela de resultados. A faixa superior é para o teste de anticorpo anti-FIV, e a faixa inferior para $\mathrm{o}$ antígeno do FeLV. Ac; anticorpo; Ag: antígeno. Fontes: A $\mathrm{e}$ B -https://www.idexx.com/small-animal-health/products-and-services/snap-fiv-felv-combo-test.html; C - http://www.doctorlab.net/docs/1437418964.pdf.

Os testes foram realizados em amostras de plasma ou soro de felinos saudáveis ou doentes provenientes de um laboratório veterinário $(\mathrm{n}=$ 48) e de clínicas veterinárias $(n=130)$ da cidade do Rio de Janeiro. O laboratório veterinário que cedeu as amostras utiliza o teste $\operatorname{SNAP}^{\circledR}$ na sua rotina. Após a realização desse teste, a mesma amostra foi encaminhada para o Laboratório de Virologia Molecular da UFRJ para avaliação, utilizando-se o teste ALERE, de forma que este fosse um estudo cego. As amostras originadas de clínicas veterinárias foram 
avaliadas simultaneamente com os dois testes pelos mesmos observadores. Todos os testes foram realizados seguindo as recomendações dos fabricantes. As amostras discordantes provenientes do laboratório veterinário foram novamente testadas com o teste $\mathrm{SNAP}^{\circledR}$ para avaliar possíveis erros de leitura do primeiro observador. Todas as amostras discordantes entre os testes rápidos ou com resultado fraco-positivo foram avaliadas por um segundo leitor. A qPCR foi utilizada como método confirmatório, seguindo o protocolo descrito por Medeiros et al. (2016) para o FIV e o protocolo descrito por Tandon et al. (2005) para o FeLV. Para a realização da qPCR, o RNA das amostras foi extraído utilizando-se o QIAamp ${ }^{\circledR}$ Viral RNA Mini Kit (Qiagen), seguindo as recomendações do fabricante. O cDNA foi obtido com o High Capacity cDNA Reverse Transcription Kit (Thermo Fisher Scientific), e a qPCR foi realizada com o sistema TaqMan®. Este estudo foi aprovado pelo Comitê de Ética em Pesquisa da UFRJ (número 150/15).

Os resultados obtidos foram submetidos à análise estatística usando-se o teste de McNemar para determinar a existência de diferença significativa entre os TRs. A sensibilidade de cada TR foi calculada dividindo-se o número de verdadeiropositivos - VP (positivos nos dois TRs e na qPCR) pelo número de VP + o número de falsonegativos - FN (negativo no TR; positivo na qPCR). A especificidade de cada TR foi calculada pela divisão do número de verdadeiro- negativos - VN (negativos nos dois TRs e na qPCR) pelo número de $\mathrm{VN}+$ o número de falsopositivos - FP (positivo no TR; negativo na qPCR). $\mathrm{O}$ intervalo de confiança e valores preditivos foram calculados.

\section{RESULTADOS}

Considerando-se os resultados concordantes entre os TRs e a qPCR, 19 amostras $(10,67 \%)$ foram positivas para FIV, $33(18,53 \%)$ foram positivas para FeLV e seis $(3,37 \%)$ foram positivas para ambos os vírus. Cento e vinte animais foram negativos para ambos os vírus $(67,41 \%)$.

Para o FeLV, resultado falso-positivo ocorreu em cinco amostras com o kit SNAP $^{\circledR}$ e em três amostras com o kit ALERE, e uma amostra apresentou resultado falso-negativo com o kit ALERE. Três amostras apresentaram resultado negativo em ambos os TRs e resultado positivo na qPCR, totalizando 12 amostras discordantes. Para o FIV, três amostras apresentaram resultados discordantes: uma com resultado falso-negativo e duas com resultado falsopositivo, todas com o kit ALERE, considerandose a qPCR como método confirmatório (Tab.1).

$\mathrm{Na}$ maioria desses casos, os resultados foram considerados fraco-positivos, pois apresentavam uma alteração de cor fraca na janela de resultado (Fig. 2).

Tabela 1. Amostras discordantes entre os dois TRs, com o resultado da qPCR

\begin{tabular}{ccccc} 
& SNAP® & ALERE & qPCR & TOTAL \\
\hline \multirow{3}{*}{ FeLV } & Negativo & Positivo & Negativo & 3 \\
& Positivo & Negativo & Negativo & 5 \\
& Positivo & Negativo & Positivo & 1 \\
Negativo & Negativo & Positivo & 3 \\
& Negativo & Positivo & Negativo & 2 \\
\hline
\end{tabular}



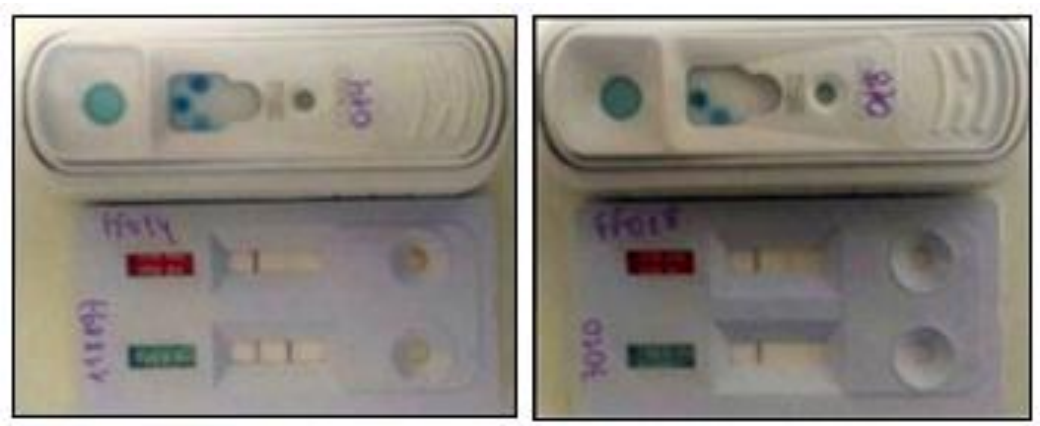

Figura 2. Amostras com resultado considerado fraco-positivo para o FIV, devido à formação de linha fraca na janela dos resultados. Nas duas situações, o resultado foi concordante entre os dois testes, porém a visualização foi mais nítida no teste $\mathrm{SNAP}^{\circledR}$.

Entre as amostras enviadas pelos clínicos, três amostras negativas para o FeLV nos dois TRs foram submetidas à qPCR para o FeLV devido à forte suspeita da infecção pelo clínico, e o resultado para todas foi positivo na qPCR (Tab. 1). Desse modo, embora os TRs tenham sido concordantes, essas amostras foram consideradas como falso-negativas. Uma das amostras de soro enviada pelo laboratório veterinário apresentou resultado positivo para FeLV no teste ALERE e negativo no SNAP ${ }^{\circledR}$. Uma amostra de plasma proveniente da mesma coleta foi enviada para efetuar nova análise, e o resultado foi negativo em ambos os TRs, sendo também negativa na qPCR. Essa amostra foi caracterizada como resultado falso-positivo no teste ALERE, visto que os resultados foram divergentes entre os TRs com o mesmo tipo de amostra (soro).

$\mathrm{O}$ teste $\mathrm{SNAP}^{\circledR}$ apresentou sensibilidade e especificidade de $100 \%$ para FIV, comparado à qPCR. Para FeLV, o teste ALERE apresentou maior especificidade e maior valor preditivo positivo em relação ao teste $\mathrm{SNAP}^{\circledR}$. Não foi observada diferença estatística entre os testes (Tab. 2).

Tabela 2. Sensibilidade, especificidade, valor preditivo positivo (VPP), valor preditivo negativo (VPN) e valor $\mathrm{p}$ dos TRs avaliados no estudo. IC: intervalo de confiança

\begin{tabular}{lllll}
\hline & & SNAP® & ALERE & Valor p \\
\hline \multirow{3}{*}{ FeLV } & Sensibilidade [95\% IC] & $93,02[80,94-98,54]$ & $90,70[77,86-97,41]$ & $0,5078^{*}$ \\
& Especificidade [95\% IC] & $96,30[91,57-98,79]$ & $97,78[93,64-99,54]$ & \\
& VPP [95\% IC] & $88,89[75,95-96,29]$ & $92,86[80,52-98,50]$ & \\
& VPN [95\% IC] & $97,74[93,55-99,53]$ & $97,06[92,64-99,19]$ & \\
& Sensibilidade [95\% IC] & $100[86,77-100]$ & $96,15[80,36-99,90]$ & $1,000^{*}$ \\
& Especificidade [95\% IC] & $100[97,6-100]$ & $98,68[95,33-99,84]$ & \\
& VPP [95\% IC] & $100[86,77-100]$ & $92,59[75,71-99,09]$ & \\
& VPN [95\% IC] & $100[97,6-100]$ & $99,34[96,37-99,98]$ & \\
\hline
\end{tabular}

IC: intervalo de confiança.

*Não significativo pelo teste de McNemar.

\section{DISCUSSÃO}

O diagnóstico de uma doença é o primeiro passo para o controle dela, e a correta detecção das infecções evita que medidas desnecessárias sejam tomadas, como segregação e eutanásia (Levy et al., 2008), além do abandono de animais (Baquero et al., 2017).
Os TRs são necessários para agilizar a conduta do clínico, apresentam boa sensibilidade e especificidade e, por isso, são utilizados como método de triagem de eleição (Banoo et al., 2010). As infecções pelo FIV e pelo FeLV estão disseminadas pelo Brasil e o número de casos vem aumentando progressivamente. A casuística deste trabalho mostra que $18,53 \%$ dos felinos foram reagentes para o antígeno do FeLV. Comparando com estudos anteriores, observa-se 
um aumento no número de casos na cidade do Rio de Janeiro, onde a ocorrência foi de $17,46 \%$, em 2002 (Souza et al., 2002), e de 11,52\%, no ano de 2012 (Almeida et al., 2012), utilizando-se o teste SNAP $^{\circledR}$ e a imunofluorescência, respectivamente. Em outros estados do Brasil, os dados variaram entre $32,5 \%$, em Belo Horizonte (Teixeira et al., 2007), e 0,33\%, em Araçatuba, São Paulo, ambos também utilizando o teste SNAP $^{\circledR}$ (Vicente Sobrinho et al., 2011). Em relação ao FIV, os dados do presente estudo foram de $10,67 \%$ de felinos soropositivos, o que demonstrou uma queda em relação ao estudo anterior realizado no Rio de Janeiro, onde a porcentagem de animais soropositivos para FIV avaliados pelo teste SNAP $^{\circledR}$ foi de $16,66 \%$ (Souza et al., 2002) avaliados pelo teste $\mathrm{SNAP}^{\circledR}$. Em relação às demais localidades do país, a soropositividade para FIV variou de $4,14 \%$, em Belo Horizonte, em 2007 (Teixeira et al., 2007), a 34,34\%, em São Paulo (Santos et al., 2013), utilizando-se o teste SNAP ${ }^{\circledR}$.

No presente estudo, dois TRs disponíveis no mercado brasileiro foram avaliados. A qPCR foi utilizada como padrão-ouro em todas as amostras. Não foi observada diferença estatisticamente significante entre os dois testes, entretanto o teste SNAP $^{\circledR}$ apresentou melhor desempenho para FIV em comparação com o teste ALERE. Os testes aqui avaliados detectam anticorpos contra diferentes proteínas do FIV (Westman et al., 2015), podendo influenciar na melhor performance do teste SNAP $^{\circledR}$. Somado a isso, o teste SNAP $^{\circledR}$ possui uma etapa de lavagem, o que aumenta a especificidade, e a amostra passa por um fluxo bidirecional, aumentando a chance de ligação antígenoanticorpo.

Em relação ao FeLV, o maior número de resultados falso-positivos foi observado no teste SNAP $^{\circledR}(5 / 6)$, dado similar ao encontrado por outros autores (Westman et al., 2016); já o teste ALERE apresentou maior especificidade em relação ao teste $\mathrm{SNAP}^{\circledR}$. Os resultados discordantes entre os métodos ocorreram principalmente nas amostras com resultado fraco-positivo, e estes foram mais facilmente visualizados no teste $\mathrm{SNAP}^{\circledR}$ para ambos os vírus. Nessas situações, a interpretação do resultado é duvidosa. Na casuística do presente estudo, nenhum dos TRs detectou resultado positivo em três amostras com suspeita clínica. As amostras foram positivas na qPCR, corroborando a necessidade de utilizar um teste confirmatório. Paralelamente a isso, um único resultado positivo para antígeno p27 do FeLV em TR é indicativo de exposição ao vírus, já que essa antigenemia pode não ser mais detectada após 12 a 16 semanas nas infecções regressivas (Hofmann-Lehmann et al., 2008).

Tendo em vista que, na infecção regressiva do FeLV, o número de anticorpos contra o vírus circulante é alto, o teste ideal para detecção dos felinos regressores seria um teste similar ao que existe para detecção do vírus da dengue, que detecta simultaneamente anticorpos das classes $\operatorname{IgM}$ e IgG, além da proteína NS1 do vírus (Osorio et al., 2010). Um teste similar para o FeLV permitiria detectar rapidamente os felinos regressores, sendo interessante para monitoramento desses felinos e para utilização em bancos de sangue, visto que os felinos regressores não deveriam ser utilizados como doadores (Nesina et al., 2015).

Em relação à execução dos TRs, o teste ALERE é de fácil execução e não necessita refrigeração, podendo ser mantido em temperatura de 2 a $30^{\circ} \mathrm{C}$. Já o teste $\mathrm{SNAP}^{\circledR}$ deve ser mantido em temperatura de 2 a $8^{\circ} \mathrm{C}$ e sua execução é um pouco mais elaborada, com maior número de etapas até a obtenção do resultado final. Sendo o Brasil um país de grandes dimensões e desigualdade socioeconômica, a possibilidade de utilização de um método de diagnóstico de fácil execução e com desempenho similar ao existente no mercado facilita o uso a campo, o que proporciona um amplo acesso, aumenta a chance de detecção dos felinos infectados e, consequentemente, o controle da disseminação dessas infecções.

\section{CONCLUSÃO}

Ambos os testes utilizados neste estudo puderam detectar satisfatoriamente a presença do FIV ou do FeLV, e as amostras de plasma geraram resultados mais precisos. No entanto, o teste confirmatório utilizando a qPCR deve ser realizado principalmente em animais com resultado de TR negativo e forte suspeita clínica de doença associada a uma dessas infecções. 


\section{AGRADECIMENTOS}

À Ana Luiza Chaves Valadão, pela revisão do manuscrito e pelos comentários; aos médicos veterinários Simone Lezan Bittencourt, Alexandra Caridade e Carlos Gabriel A. Dias, por permitirem a coleta de amostras para as análises; e à ALERE, por ceder os kits para a realização do trabalho. Este trabalho obteve apoio financeiro da Faperj. Os financiadores deste projeto não tiveram nenhum papel no desenho do estudo, coleta e análise de dados, decisão de publicar ou preparação do manuscrito.

\section{REFERÊNCIAS}

ALMEIDA, N.R.; DANELLI, M.G.M.; SILVA, L.H.P. et al. Prevalence of feline leukemia virus infection in domestic cats in Rio de Janeiro. $J$. Feline Med. Surg., v.14, p.583-586, 2012.

ARJONA, A.; ESCOLAR, E.; SOTO, I. et al. Seroepidemiological survey of infection by feline leukemia virus and immunodeficiency virus in Madrid and correlation with some clinical aspects. J. Clin. Microbiol., v.38, p.34483449, 2000

BANOO, S.; BELL, D.; BOSSUYT, P. et al. Evaluation of diagnostic tests for infectious diseases: general principles. Nat. Rev. Microbiol., v.8, p.S17-S29, 2010.

BAQUERO, O.S.; CHIOZZOTTO, E.N.; GARCIA, R.C.M. et al. Abandonment of dogs and cats: public opinions as population management indicators. J. Appl. Anim. Welf. Sci., v.26, p.1-7, 2017.

DANDEKAR, S.; BEEBE, A.M.; BARLOUGH, $\mathrm{J}$. et al. Detection of feline immunodeficiency virus (FIV) nucleic acids in FIV-seronegative cats. J. Virol., v.66, p.4040-4049, 1992.

DIEHL, L.J.; MATHIASON-DUBARD, C.K.; O'NEIL, L.L.; HOOVER, E.A. Plasma viral RNA load predicts disease progression in accelerated feline immunodeficiency virus infection. J. Virol., v.70, p.2503-2507, 1996.

GOMES-KELLER, M.A.; TANDON, R.; GÖNCZI, E. et al. Shedding of feline leukemia virus RNA in saliva is a consistent feature in viremic cats. Vet. Microbiol., v.1, p.11-21, 2006.
HARTMANN, K. Clinical aspects of feline retroviruses: a review. Viruses, v.31, p.26842710, 2012.

HOFMANN-LEHMANN, R.; CATTORI, V.; TANDON, R. et al. How molecular methods change our views of FeLV infection and vaccination. Vet. Immunol. Immunopathol., v.123, p.119-123, 2008.

HOSIE, M.J.; ADDIE, D.; BELÁK, S. et al. Feline immunodeficiency ABCD guidelines on prevention and management. J. Feline Med. Surg., v.11, p.575-584, 2009.

LEVY, J.; CRAWFORD, C.; HARTMANN, K. et al. American Association of Feline Practitioners' feline retrovirus management guidelines. J. Feline Med. Surg., v.3, p.300-316, 2008.

MATTEUCCI, D.; BALDINOTTI, F.; MAZZETTI, P. et al. Detection of feline immunodeficiency virus in saliva and plasma by cultivation and polymerase chain reaction. J. Clin. Microbiol., v.31, p.494-501, 1993.

MEDEIROS, S.O.; ABREU, C.M.; DELVECCHIO, R. et al. Follow-up on long-term antiretroviral therapy for cats infected with feline immunodeficiency virus. J. Feline Med. Surg., v.18, p.264-272, 2016.

NESINA, S.; KATRIN HELFERHUNGERBUEHLER, A.; RIOND, B. et al. Retroviral DNA-the silent winner: blood transfusion containing latent feline leukemia provirus causes infection and disease in naïve recipient cats. Retrovirology, v.12, p.1, 2015.

OBERT, L.A.; HOOVER, E.A. Early pathogenesis of transmucosal feline immunodeficiency virus infection. J. Virol., v.76, p.6311-6322, 2002.

OSORIO, L.; RAMIREZ, M.; BONELO, A. et al. Comparison of the diagnostic accuracy of commercial NS1-based diagnostic tests for early dengue infection. Virol. J., v.7, p.1, 2010.

PAILLOT, R.; RICHARD, S.; BLOAS, F.; PIRAS, F. et al. Toward a detailed characterization of feline immunodeficiency vírus-specific $\mathrm{T}$ cell imune responses and mediated imune disorders. Vet. Immunol. Immunopathol., v.15, p.1-14, 2005. 
RAVAZZOLO, A.P.; COSTA, U.M. Retroviridae. In: FLORES, E.F. (Ed.). Virologia veterinária. Santa Maria: UFSM, 2007. p.955-985.

SANTOS, D.L.; LUCAS, R.; LALLO, M.A. Epidemiologia da imunodeficiência viral, leucemia viral e peritonite infecciosa em felinos procedentes de um hospital veterinário. Rev. Acad. Ciênc. Agrár. Ambient., v.11, p.161-168, 2013.

SOUZA, H.J.M.; TEIXEIRA, C.H.R.; GRAÇA, R.F.S. Estudo Epidemiológico das infecções pelos vírus da leucemia e/ou imunodeficiência felina em gatos domésticos do município do Rio de Janeiro. Clín. Vet., v.7, p.14-21, 2002.

TANDON, R.; CATTORI, V.; GOMESKELLER, M.A. et al. Quantitation of feline leukaemia virus viral and proviral loads by TaqMan real-time polymerase chain reaction. $J$. Virol. Methods, v.130, p.124-132, 2005.

TEIXEIRA, B.M.; RAJÃO, D.S.; HADDAD, J.P.A. et al. Ocorrência do vírus da imunodeficiência felina e do vírus da leucemia felina em gatos domésticos mantidos em abrigos no município de Belo Horizonte. Arq. Bras. Med. Vet. Zootec., v.59, p.939-942, 2007.
TORRES, A.N.; MATHIASON, C.K.; HOOVER, E.A. Re-examination of feline leukemia virus: host relationships using real-time PCR. Virology, v.332, p.272-283, 2005.

VICENTE SOBRINHO, L.S.; VIDES, J.P.; BRAGA, E.T. et al. Sorofrequência de infecção pelo vírus da imunodeficiência felina e vírus da leucemia felina em gatos do município de Araçatuba, São Paulo. Braz. J. Vet. Res. Anim. Sci., v.48, p.378-383, 2011.

WESTMAN, M.E.; MALIK, R.; HALL, E. et al. Determining the feline immunodeficiency virus (FIV) status of FIV-vaccinated cats using pointof-care antibody kits. Comp. Immunol. Microbiol. Infect. Dis., v.42, p.43-52, 2015.

WESTMAN, M.E.; MALIK, R.; HALL, E.; NORRIS, J.M. Diagnosing feline immunodeficiency virus (FIV) infection in FIVvaccinated and FIV-unvaccinated cats using saliva. Comp. Immunol. Microbiol. Infect. Dis., v.46, p.66-72, 2016. 